\title{
The Character Table of an Eight-Dimensional Orthogonal Group
}

\author{
By David C. Hunt
}

\begin{abstract}
This paper describes the calculation of the character table of the 8-dimensional orthogonal group of maximal index over the field with 3 elements. The group is of interest as it is a subgroup of relatively small index in the sporadic simple group $M(23)$ defined by $B$. Fischer [2]. The group also has an outer automorphism group of order 24 , isomorphic to the symmetric group on 4 symbols.
\end{abstract}

1. Introduction. Let $O^{+}(n, q), n$ even, denote the group of orthogonal $n \times n$ matrices of maximal Witt index $n / 2$ over the field $G F(q)$.

The order of $O^{+}(8,3)$ is $2^{15} \cdot 3^{12} \cdot 5^{2} \cdot 7 \cdot 13 . O^{+}(8,3)$ has a subgroup $S \Omega^{+}(8,3)$ of index 4. The centre of $S \Omega^{+}(8,3)$ is of order 2 and the factor group $P S \Omega^{+}(8,3)$ is a simple group of order $2^{12} \cdot 3^{12} \cdot 5^{2} \cdot 7 \cdot 13$. $P S \Omega^{+}(8,3)$ is the simple Chevalley group known as $D_{4}(3)$.

2. Conjugacy Classes of $\operatorname{PS\Omega }^{+}(8,3)$. A theorem of Wall [4] gives the conjugacy classes in $O^{+}(8,3)$. Those conjugacy classes containing elements of determinant 1 and which belong to the kernel of the spinorial norm lie in $S \Omega^{+}(8,3)$. Some of those classes split into 2 or 4 conjugacy classes in $S \Omega^{+}(8,3)$. The conjugacy classes in $P S \Omega^{+}(8,3)$ come from forming the factor group of $S \Omega^{+}(8,3)$ by its centre. $P S \Omega^{+}(8,3)$ has 113 conjugacy classes and they are listed in Table 1 on the microfiche supplement.

3. Permutation Representations of Small Degree. $P S \Omega^{+}(8,3)$ acts intransitively on the projective geometry $P G(8,3)$ which consists of $\left(3^{8}-1\right) /(3-1)=$ 3280 points. The group acts transitively on the points of lengths 0,1 and 2 , respectively. This gives a rank 3 permutation representation of degree 1120 and 2 different rank 3 permutation representations of degree 1080 . The values of the corresponding permutation characters can easily be calculated for every conjugacy class in $P S \Omega^{+}(8,3)$. The values of 2 of these permutation characters are given in columns of Table 1. The stabilizer of a point in the representation on 1080 points is $P S \Omega(7,3)$. The character table of $P S \Omega(7,3)$ has appeared in an earlier paper by the author (Hunt [3]). It is possible to determine how the conjugacy classes of $P S \Omega(7,3)$ fuse as a subgroup of $P S \Omega^{+}(8,3)$. Hence, it is possible to restrict the above permutation representations to $\operatorname{PS\Omega }(7,3)$ and split them into irreducible characters of $\operatorname{PS} \Omega(7,3)$. This set of irreducible characters partitions into 3 subsets which are the components of the 3 irreducible characters of $P S \Omega^{+}(8,3)$ restricted to $\operatorname{PS\Omega }(7,3)$. Hence, it is possible to

Received November 13, 1972.

AMS (MOS) subject classifications (1970). Primary 20C15, 20D05; Secondary 20H30. 
calculate the values of the characters of degree $1,300,260,819$ on most of the conjugacy classes and the remaining values are uniquely determined on all conjugacy classes of $P S \Omega^{+}(8,3)$.

4. The Character Table. Applying the automorphism group to the irreducible characters determined above yields 11 irreducible characters. A large number of generalized characters can be determined by forming tensor products and symmetric and alternating products of the known irreducible characters. Other generalized characters can be determined by inducing characters from subgroups isomorphic to $P S \Omega(7,3)$ and $P S \Omega^{+}(8,2)$. The character table of $P S \Omega^{+}(8,2)$ can be found in Dye [1]. The permutation representation on the cosets of a subgroup isomorphic to $P S \Omega^{+}(8,2)$ is given as the final column of Table $1 . P S \Omega^{+}(8,3)$ contains at least 4 conjugacy classes of subgroups isomorphic to $P S \Omega^{+}(8,2)$. The above generalized characters are sufficient to determine the entire rational character table; see Table 2 on the microfiche supplement. Only one rational character is not absolutely irreducible, the last of the 113 which is the sum of 2 absolutely irreducible characters.

Acknowledgement. The author thanks Professor D. Livingstone and Mr. E. W. Wynn of the University of Birmingham, England, for their assistance. In particular, Mr. Wynn's help with computer calculations is acknowledged.

Department of Mathematics

The University of New South Wales

Kensington, 2033, N.S.W., Australia

1. R. H. DYE, "The simple group $F H(8,2)$ of order $212 \cdot 35 \cdot 52 \cdot 7$ and the associated geometry of triality," Proc. London Math. Soc. (3), v. 18, 1968, pp. 521-562. MR 37 \#1468.

2. B. FISCHER, "Finite groups generated by 3-transpositions. I," Invent. Math., v. 13, 1971, pp. 232-246. MR 45 \# 3557.

3. D. C. HuNT, "Character tables of certain finite simple groups," Bull. Austral. Math. Soc., v. 5, 1971, pp. 1-42. MR 46 \#1896.

4. G. E. WALL, "On the conjugacy classes in the unitary, symplectic and orthogonal groups," J. Austral. Math. Soc., v. 3, 1963, pp. 1-62. MR 27 \#212.

\title{
The Character Table of Fischer's Simple Group, $M(23)$
}

\author{
By David C. Hunt
}

Abstract. This paper describes the calculation of the character table of $M(23)$, the sporadic simple group discovered by B. Fischer [1].

Received November 13, 1973.

AMS (MOS) subject classifications (1970). Primary 20C15, 20D05; Secondary $20 \mathrm{H} 30$. 